Article type : Therapeutic Vignette

Short title running head: Therapeutic vignette

Authors running head: Therapeutic vignette

Running section head: Therapeutic vignette

Correspondence:Dr M. Vu. Dermatology Department, The Royal Melbourne Hospital, 300 Grattan Street,

Parkville, Victoria, Australia, 3050

E-mail: hoa-mi.vu@mh.org.au

Conflict of interest: the authors declare that they have no conflicts of interest.

Accepted for publication 16 January 2017

CPD • Therapeutic vignette

\title{
Oral tofacitinib: a promising treatment in atopic dermatitis, alopecia areata and vitiligo
}

\author{
M. Vu, ${ }^{1}$ C. Heyes, ${ }^{1}$ S. J. Robertson, ${ }^{1-4}$ G. A. Varigos ${ }^{1}$ and G. Ross ${ }^{1}$ \\ ${ }^{1}$ Department of Dermatology, Royal Melbourne Hospital, Victoria, Australia,'Department of Dermatology, The Royal Children's \\ Hospital, Melbourne, Australia; ${ }^{3}$ Department of Dermatology, Monash Health, Melbourne, Australia; and ${ }^{4}$ Murdoch Childrens Research \\ Institute, Melbourne, Australia
}

Tofacitinib citrate is a Janus kinase (JAK)-1/3 inhibitor from a novel therapeutic class of medications used for systemic inflammatory disorders such as rheumatoid arthritis. However, its use in dermatology has not been well explored.

JAK inhibitors may be of benefit in treating atopic dermatitis (AD) by downregulating the exaggerated Thelper (Th)2 immune response. Interleukin (IL)-4 is crucial in promoting Th2 differentiation with release of the cytokines IL-4, $-5,-10$ and $-13 .{ }^{1}$ As IL-4 signalling is mediated by the JAK- $1 / 3$ and STAT- 6 pathways, this therapeutic target is inhibited by tofacitinib. ${ }^{1}$ In alopecia areata (AA) and vitiligo, cytotoxic CD8+NKG2D+ T cells and increased interferon (IFN) expression are implicated in disease induction. ${ }^{2}$ In AA, CD8+T-cell production of IFN- $\gamma$ leads to collapse of immune privilege and increased production of IL-15, propagating autoimmune destruction of the hair follicle. ${ }^{2}$ Within vitiligo, elevation of IFN- $\gamma$ induces expression of C-X-C motif chemokine-10 in keratinocytes, mediating depigmentation. ${ }^{3}$ As IFN- $\gamma$ signalling occurs through the JAK-STAT pathway, its effects can be downregulated by tofacitinib. ${ }^{3}$

The most common adverse effects of tofactinib include opportunistic infections, diarrhoea, headache and hypertension. ${ }^{4}$ Monitoring of fasting lipids, full blood count (FBC), urea and electrolytes (UECs), liver function tests (LFTs) and creatine kinase (CK) is necessary as tofacitinib may cause hyperlipidaemia, neutropenia, lymphopenia, anaemia and hepatitis, and elevate serum creatinine and CK levels. ${ }^{4}$

A 44-year-old white man with a lifelong history of $A D$ presented with an acute exacerbation of the disease with

This article is protected by copyright. All rights reserved 
secondary infection. He also had a 4-year history of AA (with previous alopecia totalis) and a 3-year history of nonsegmental multifocal vitiligo. The $A A$ and vitiligo had been persistent but stable during the preceding 3 months.

The infective flare of $A D$ was treated with oral antibiotics, prednisolone $50 \mathrm{mg}$, narrowband ultraviolet $B$ and betamethasone dipropionate ointment. The infective component resolved, but the AD (and also the AA and vitiligo) persisted despite 12 weeks of therapy. As our patient had three treatment-refractory conditions potentially responsive to tofacitinib, treatment was initiated with tofacitinib $5 \mathrm{mg}$ twice daily over 6 months. Concurrent treatment included prednisolone $5 \mathrm{mg}$ daily and betamethasone dipropionate ointment. The patient unerwetn clinical review fortnightly for 8 weeks and monthly thereafter, with Eczema Area and Severity Index (EASI) and Vitiligo Area Scoring Index (VASI) determined at each review. Monthly monitoring of fasting lipids, FBC, UECs, LFTs and CK was performed with no abnormality detected.

At baseline, the patient had scattered eczematous plaques and depigmented macules of vitiligo on his trunk and extremities. There were well-circumscribed patches of AA on the scalp and beard region and almost complete absence of hair on the eyebrows, eyelashes, extremities and axillae. Complete depigmentation of the scalp and body hair was observed.

The 6-month trial of oral tofacitinib demonstrated clinical response in all three conditions. The AD demonstrated the most significant improvement, with a decline in EASI from 14.4 at baseline to 11.7 with 2 weeks of treatment, and complete remission of the AD (EASI 0.0) with 3 months of treatment (Fig. 1). Prednisolone $5 \mathrm{mg}$ was ceased after 6 weeks of tofacitinib therapy, and topical betamethasone dipropionate usage reduced from $35 \mathrm{~g}$ per week at commencement to $2 \mathrm{~g}$ per week at the 3-month follow-up.

The patient's AA responded after 1 month, with continued slow improvement throughout the treatment course. There was reduction of the AA on the scalp and beard region, with increased hair growth on the extremities, eyebrows and eyelashes (Fig. 2).

There was only marginal improvement in vitiligo, with a decline in VASI score from 4.68 at baseline to 3.95 at 5 months.

Tofacitinib treatment was well tolerated. There were two episodes of self-resolving upper respiratory tract infections and diarrhoea, not requiring treatment interruption.

Limited evidence exists for use of tofacitinib in $A D, A A$ and vitiligo., ${ }^{3,5,6}$ There is a single case series of six patients treated with tofacitinib for recalcitrant $A D,{ }^{5}$ which showed a statistically significant reduction in average SCOring of Atopic Dermatitis (SCORAD) score from 36.5 to 16.5 at $4-14$ weeks $(P<0.05)$ and 36.5 to 12.2 at 829 weeks $(P<0.05)$ of treatment mwith a similar decline in pruritus and sleep assessment scores. ${ }^{5}$

Craiglow et al. reported the first successful use of tofacitinib in vitiligo ${ }^{3}$ and AA. ${ }^{6}$ In a patient with widespread progressive vitiligo, there was a clinical response at 2 months and almost complete repigmentation of the forehead and hands at 5 months, ${ }^{3}$ while for $A A,{ }^{6}$ almost complete regrowth of hair at all sites was achieved within 8 months of treatment.

In our case, the AD had the greatest reponse; however, the clinical remission cannot be solely attributed to tofacitinib as the patient was aksi on conventional treatment. Although we cannot exclude a natural course of recovery of AA and vitiligo, the patient's history of stable disease prior to commencement of treatment supports the likely influence of tofacitinib on these two conditions. Despite different pathomechanisms of disease, this case demonstrates that clinical response is possible with single-agent tofacitinib.

Our report adds to the growing evidence to support use of tofacitinib in $A D, A A$ and perhaps vitiligo. Randomized controlled studies are needed to validate its efficacy in these dermatological conditions. 


\section{References}

1. Bao L, Zhang H, Chan L. The involvement of the JAK-STAT signalling pathway in chronic inflammatory skin disease atopic dermatitis. JAKSTAT 2013; 2: e24137.

2. Harris J. Vitiligo and alopecia areata: Apples and oranges? Exp Dermatol 2013; 22: 785-89.

3. Craiglow B, King B. Tofacitinib citrate for the treatment of vitiligo: a pathogenesis directed-therapy. JAMA Dermatol 2015; 151: 1110-12.

4. Xeljanz product monograph. Canada: Pfizer, 2015. Available from: http://www.pfizer.ca/sites/g/files/g10028126/f/201511/Xeljanz_PM_E.pdf (accessed 27 December 2016].

5. Levy L, Urban J, King B. Treatment of recalcitrant atopic dermatitis with the oral Janus kinase inhibitor tofacitinib citrate. J Am Acad Dermatol 2015; 73: 395-9.

6. Craiglow B, King B. Killing two birds with one stone: oral tofacitinib reverses alopecia universalis in a patient with plaque psoriasis. J Invest Dermatol 2014; 134: 2988-90.

Figure 1 Atopic dermatitis on the trunk at (a) baseline with a corresponding Eczema Area and Severity Index (EASI) of 14.4 and (b) 3 months with corresponding EASI of 0.0 following oral tofacitinib use.

Figure 2 Alopecia areata at (a) baseline with patchy alopecia on the scalp, loss of eyebrows and eyelashes, and (b) regrowth evident at 6 months following oral tofacitinib use.

\section{CPD questions}

\section{Learning objective}

To explore the use of oral tofacitinib in the treatment of atopic dermatitis, alopecia areata and vitiligo.

\section{Question 1}

Oral tofacitinib may be effective for the treatment of alopecia areata and vitiligo through which of the following mechanisms?

(a) Downregulating the T helper 2 immune response.

(b) Inhibiting CD4+ lymphocytes.

(c) Downregulating interferon(IFN) $\gamma$.

(d) Inhibiting natural killer (NK) cells.

(e) Inhibiting interleukin (IL)-17.

\section{Question 2}

Which of the following laboratory parameters should be monitored during tofacitinib treatment?
(a) Fasting lipids
(b) Fasting glucose.
(c) Uric acid.
(d) Fasting insulin.
(e) $\mathrm{HbA} 1 \mathrm{c}$. 


\section{Answer 1}

Oral tofacitinib may be effective for the treatment of alopecia areata and vitiligo through which of the following mechanisms?

(a) Incorrect. Downregulation of theTh2 immune response is the mechanism of action of tofacitinib in atopic dermatitis.

(b) Incorrect. CD4+ lymphocytes are not inhibited by tofacitinib.

(c) Correct. IFN- $\gamma$ is implicated in the pathogenesis of alopecia areata and vitiligo, and its effect is downregulated by tofacitinib.

(d) Incorrect. NK cells are not inhibited by tofacitinib.

(e) Incorrect. IL-17 is not inhibited by tofacitinib.

\section{Answer 2}

Which of the following laboratory parameters should be monitored during tofacitinib treatment?

(a) Correct. Fasting lipids should be monitored during tofacitinib treatment as hyperlipidaemia has been reported as an adverse effect.

(b) Incorrect. Deranged blood glucose levels have not been reported as an adverse effect of tofacitinib.

(c) Incorrect. Deranged uric acid levels have not been reported as an adverse effect of tofacitinib.

(d) Incorrect. Altered fasting insulin levels have not been reported as an adverse effect of tofacitinib.

(e) Incorrect. Altered $\mathrm{HbA} 1 \mathrm{c}$ has not been reported as an adverse effect of tofacitinib.

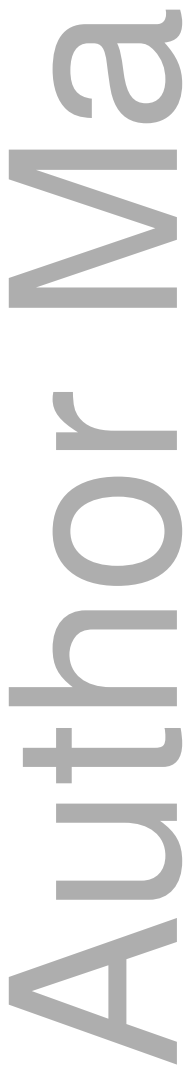


a)

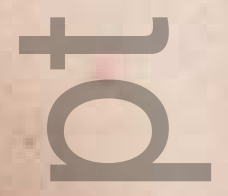

D
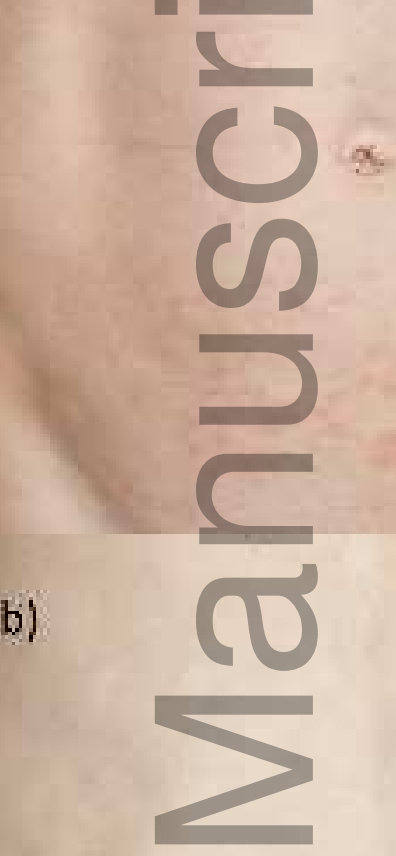

b)
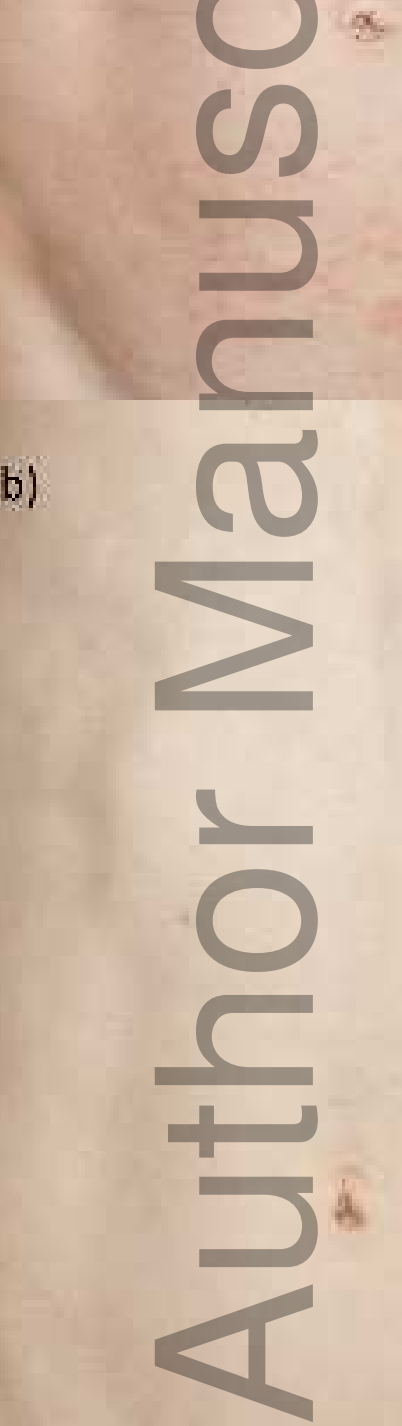


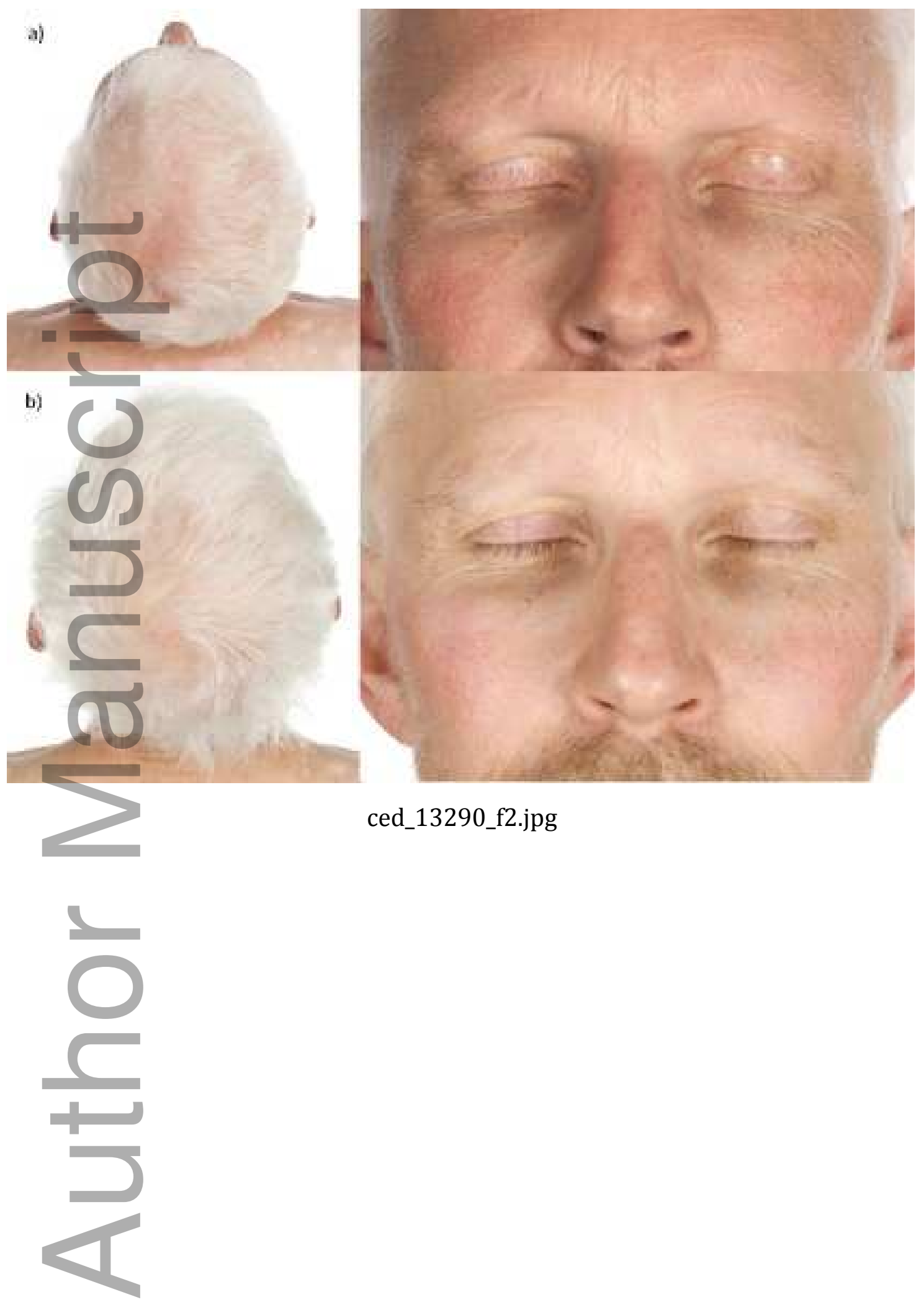

This article is protected by copyright. All rights reserved 


\section{University Library}

\section{- M M N E R VA A gateway to Melbourne's research publications}

Minerva Access is the Institutional Repository of The University of Melbourne

Author/s:

Vu, M;Heyes, C;Robertson, SJ;Varigos, GA;Ross, G

Title:

Oral tofacitinib: a promising treatment in atopic dermatitis, alopecia areata and vitiligo

Date:

2017-12-01

Citation:

Vu, M., Heyes, C., Robertson, S. J., Varigos, G. A. \& Ross, G. (2017). Oral tofacitinib: a promising treatment in atopic dermatitis, alopecia areata and vitiligo. CLINICAL AND EXPERIMENTAL DERMATOLOGY, 42 (8), pp.942-944. https://doi.org/10.1111/ced.13290.

Persistent Link:

http://hdl.handle.net/11343/293583 